\title{
Abundance analysis of two late A-type stars HD 32115 and HD 37594 $4^{\star \star \star}$
}

I. F. Bikmaev ${ }^{1}$, T. A. Ryabchikova ${ }^{2,3}$, H. Bruntt ${ }^{4}$, F. A. Musaev $^{5,6,7}$, L. I. Mashonkina ${ }^{1}$, E. V. Belyakova ${ }^{1}$, V. V. Shimansky ${ }^{1}$, P. S. Barklem ${ }^{8}$, and G. Galazutdinov ${ }^{5,6}$

1 Department of Astronomy, Kazan State University, Kremlevskaya 18, 420008 Kazan, Russia e-mail: Ilfan.Bikmaev@ksu.ru

2 Institute of Astronomy, Russian Academy of Sciences, Pyatnitskaya 48, 109017 Moscow, Russia

3 Institute for Astronomy, University of Vienna, Türkenschanzstrasse 17, 1180 Vienna, Austria e-mail: ryabchik@tycho.astro.univie.ac.at

4 Institute for Physics and Astronomy, University of Aarhus, Bygn. 520, 8000 Aarhus C, Denmark e-mail: bruntt@ifa.au.dk

5 Special Astrophysical Observatory RAS, 369167 Nizhnij Arkhyz, Karachai-Circassian Republic, Russia e-mail: faig@sao.ru

6 SAO Branch of Isaac Newton Institute, Santiago, Chile

7 International Centre for Astronomical, Medical and Ecological Researches (ICAMER), National Academy of Sciences of Ukraine, 361605 Peak Terskol, Kabardino-Balkaria, Russia

8 Department of Astronomy and Space Physics, Uppsala University, Box 515, 751-20 Uppsala, Sweden e-mail: barklem@astro.uu.se

Received 26 March 2002 / Accepted 9 April 2002

\begin{abstract}
We have performed abundance analysis of two slowly rotating, late A-type stars, HD 32115 (HR 1613) and HD 37594 (HR 1940), based on obtained echelle spectra covering the spectral range 4000-9850 A. These spectra allowed us to identify an extensive line list for 31 chemical elements, the most complete to date for A-type stars. Two approaches to abundance analysis were used, namely a "manual" (interactive) and a semi-automatic procedure for comparison of synthetic and observed spectra and equivalent widths. For some elements non-LTE (NLTE) calculations were carried out and the corresponding corrections have been applied. The abundance pattern of HD 32115 was found to be very close to the solar abundance pattern, and thus may be used as an abundance standard for chemical composition studies in middle and late A stars. Further, its $\mathrm{H} \alpha$ line profile shows no coreto-wing anomaly like that found for cool Ap stars and therefore also may be used as a standard in comparative studies of the atmospheric structures of cool, slowly rotating Ap stars. HD 37594 shows a metal deficiency at the level of -0.3 dex for most elements and triangle-like cores of spectral lines. This star most probably belongs to the $\delta$ Sct group.
\end{abstract}

Key words. stars: atmospheres - stars: abundances - stars: individual: HD 32115, HD 37594

\section{Introduction}

Solar photospheric abundances are usually used as a reference for any abundance study. However, it has been shown that the mean abundances for some elements obtained for large homogeneous groups of stars can differ

Send offprint requests to: T. Ryabchikova,

e-mail: ryabchik@inasan.rssi.ru

* Based on observations obtained at the 2-m telescope of Peak Terskol Observatory near Elbrus mountain, International Center of Astronomical and Medical-Ecological Researches, Russia.

** Table 4 is only available in electronic form at http://www.edpsciences.org significantly from solar abundances. For example, Luck \& Lambert $(1981,1985)$ and later Takeda \& Takada-Hidai (1998) obtained an oxygen deficiency of 0.3 dex relative to the Sun for a large group of A to K supergiants. There are some indications that the sun may be slightly ironrich (+0.09 dex on average) as compared to solar-like stars of the same age (Gustafsson 1998). Extensive abundance studies of the superficially normal sharp-lined Band F-type dwarfs were performed by S. Adelman and coworkers (see Adelman et al. 2000 and references therein). They found that many of the investigated stars show Amtype phenomena, and only 5 stars with effective temperatures in the range $6700-9000 \mathrm{~K}$ have abundances close to the solar photospheric values. Their study was performed 
Table 1. Observational $\log$ for HD 32115 and HD 37594. Radial velocity measurements based on $n$ lines with standard deviations in units of the last digit (in parentheses) are given in the second column.

\begin{tabular}{cccc}
\hline \hline HJD-2400000 & $V_{\mathrm{r}, \mathrm{km} \mathrm{s}^{-1}} n$ & Reference \\
\hline 52333.2007 & $19.15(43)$ & 67 & this paper \\
51929.3035 & $42.08(45)$ & 71 & this paper \\
49405.5333 & $44.61(43)$ & & Grenier et al. (1999) \\
42675.8313 & $43.7(5)$ & 14 & Nordström \& Andersen (1985) \\
42669.8640 & $12.2(4)$ & 13 & Nordström \& Andersen (1985) \\
42379.7278 & $6.8(6)$ & 14 & Nordström \& Andersen (1985) \\
& & HD 37594 & this paper \\
51930.2146 & $22.52(72)$ & 69 & Grenier et al. (1999) \\
49736.6142 & $22.04(39)$ & & Nordström \& Andersen (1985) \\
42680.8220 & $22.4(3)$ & 15 & Nordström \& Andersen (1985) \\
42675.8928 & $24.8(6)$ & 16 & Nordström \& Andersen (1985) \\
42380.6909 & $20.2(7)$ & 16 &
\end{tabular}

mainly in the blue spectral region (up to $5000 \AA$ ), and therefore abundances of C, N, O, Na, S, K were absent or rather questionable. Varenne \& Monier (1999) published abundances for $48 \mathrm{~A}$ - and F-type dwarfs in the Hyades open cluster. They have only two stars hotter than $6650 \mathrm{~K}$ with $v \sin i \leq 25 \mathrm{~km} \mathrm{~s}^{-1}$. Because of the limited spectral regions that were observed abundances for only 11 elements have been derived. Half of these abundances were based on 1 or 2 spectral lines.

Most sharp-lined stars with temperatures in the range 6700-8000 K belong to different groups of peculiar stars: Ap, roAp, Am, or $\lambda$ Boo groups. They are usually believed to have the same or nearly the same atmospheric structure as normal stars. However, Cowley et al. (2001) found a pronounced core-to-wing anomaly in the Balmer lines of some Ap stars. They could not compare the $\mathrm{H} \alpha$ line profiles of these stars with normal solar abundance stars because of the lack of reliable spectroscopic standards in this temperature region. We therefore decided to perform an accurate spectroscopic investigation of sharp-lined late A- to early F-type stars that are classified as normal MS stars, using observations of a wide spectral region 4000-9850 A. From tabulated rotational velocities (Abt \& Morrell 1995) we extracted 27 stars classified as normal A3-F5-type stars of luminosity classes III-V with $v \sin i \leq$ $25 \mathrm{~km} \mathrm{~s}^{-1}$. Among them only 13 stars have metallicities in the range $|[M]| \leq 0.15$ on the basis of temperaturegravity-metallicity calibrations of the observed Strömgren colours (a procedure which will be described below). We did not consider the remainder of the stars, the majority of which have low metallicities $[M]<-0.15$. In this paper we present the results of careful atmospheric parameter and abundance determinations for two stars: HD 32115 and HD 37594. These stars were classified as A8IV (HD 32115) and A8Vs (HD 37594) by Cowley et al. (1969).

\section{Observations and data reduction}

High resolution spectra of the region 4000-9850 $\AA$ were obtained for both stars with the coude-echelle spectrometer (Musaev et al. 1999) mounted on the $2 \mathrm{~m}$ "Zeiss" telescope at Peak Terskol Observatory near Elbrus mountain in Russia. The best resolving power of the spectrograph in this operational mode is $R=45000$, but because of the rather poor seeing we used a wider slit that resulted in a reduced spectral resolution of $R \approx 30000$. A Wright Instruments front-side illuminated CCD of 1242 by 1152 pixels $(22 \mathrm{mkm})$ was used as a detector for simultaneous registration of the whole spectral range in a single exposure. Two spectra were obtained for each star and they were coadded after cleaning for cosmic events during the reduction procedure. Blueward of $7100 \AA$ the consecutive orders overlap, while there are gaps of 5 to $55 \AA$ between the orders for $\lambda \geq 7100 \AA$. Typical $S / N=$ 150 was estimated for the centers of the orders close to the $\mathrm{H} \alpha$ region, while $S / N$ decreases to about 100 in the blue and infrared regions. Note that $S / N$ also decreases from the center to both ends of the order. An extra spectrum of HD 32115 was obtained recently and was used for radial velocity measurements, and for equivalent widths measurements of a few lines in the infrared spectral region. Table 1 contains the Heliocentric Julian Dates of the middle of the exposure for each pair of observations. The second column of Table 1 presents the results of the radial velocity measurements (see below) together with the data from the literature.

Spectrum processing was realised with the help of a modified version of the PC-based DECH software (Galazutdinov 1992). It includes background subtraction, echelle vectors extraction from the echelleimages, wavelength calibration, continuum rectification, 
line identification, equivalent widths and radial velocity measurements. We did not divide the stellar image by a flat-field as we found this procedure does not improve the initial $S / N=100-150$ for this particular CCD.

\section{Radial velocities}

Each spectral line chosen for measurement was approximated by a Gaussian. We measured 332 and 268 lines in the spectra of HD 32115 and HD 37594 respectively. The full list of spectral lines in the 4000-9850 $\AA$ spectral region for both stars was extracted from the VALD database (Kupka et al. 1999). For the final radial velocity we have chosen only Fe I lines with the best laboratory wavelengths (Nave et al. 1994). Our $V_{\mathrm{r}}$ values and their standard deviations are given in the second column of Table 1 together with data collected from the literature. HD 37594 may be considered as a single star on the basis of six radial velocity measurements, while HD 32115 shows clear $V_{\mathrm{r}}$ variations and may be a binary system. From the range of the radial velocities presented in Table 1 we may conclude that our first observations were undertaken near the maximum possible separation between the components. Careful synthesis of the whole spectrum of HD 32115 does not reveal any trace of the lines of the secondary star, and therefore we conclude that it is most likely relatively faint and thus has no significant influence on the primary's spectrum.

\section{Atmospheric parameters}

Stellar parameters derived with different methods are given in Table 2. We first derived effective temperatures, gravities, metallicities and absolute magnitudes of our stars employing Strömgren photometric indices and the calibration of Hauck \& Mermilliod (1998). We used the TEMPLOGG procedure (Rogers 1995), which for A3-F0type stars is based on the original calibrations by Moon \& Dworetsky (1985) with improvements by Napiwotski et al. (1993). For the metallicity calibration TEMPLOGG uses a relation between the Strömgren metallicity index and the stellar metallicity derived by Smalley (1993). The quoted formal errors in the stellar parameters from photometry account only for the reported accuracy of the photometric indices. One can see excellent agreement between absolute magnitudes obtained here and those obtained from Hipparcos parallaxes (ESA 1997). In this case the formal errors in the surface gravity include both the parallax uncertainty and an effective temperature uncertainty of $\pm 100 \mathrm{~K}$. This provides strong support for the surface gravities used in our analysis. Bolometric corrections do not exceed 0.06 mag. On evolutionary tracks both stars lie close to the ZAMS and have masses slightly more than $1.5 M_{\odot}$ (Pamyatnykh et al. 1998; Pamyatnykh 2000). The age of both stars is estimated at 600-700 Myr.

To check the influence of the metal abundances on the derived atmospheric parameters we calculated a small grid of atmospheric models and corresponding colours with the

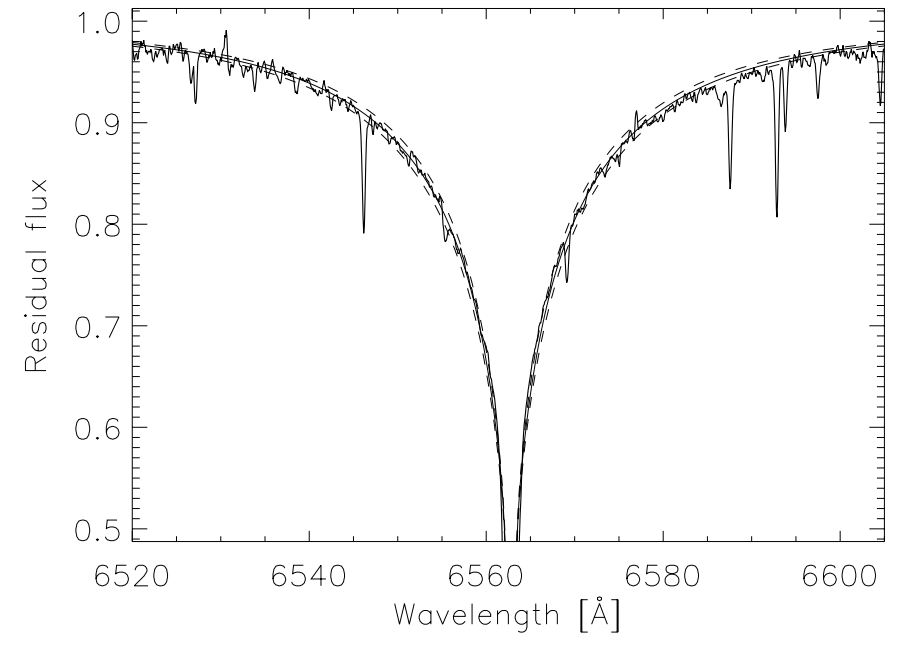

Fig. 1. A comparison between the observed $\mathrm{H} \alpha$ line profile for HD 32115 (thick full line) and those calculated for 3 atmospheric models: 7250_newODF (adopted model - thin full line), 7150_newODF (upper dashed line) and 7350_newODF.

abundances typical for our stars (see Sect. 5) and compared the observed and synthetic colours. Calculations were made with the ATLAS9 code (Kurucz 1993) which is modified by the inclusion of new line opacities in the form of specifically computed ODFs (Piskunov \& Kupka 2001) and the Canuto-Mazitelli convection treatment (Smalley \& Kupka 1997). We obtained the best fit to Strömgren indices with $T_{\text {eff }}=7250 \mathrm{~K}$ and $T_{\text {eff }}=7170 \mathrm{~K}$ for HD 32115 and HD 37594, respectively. They are given in Table 2 as ATLAS9-newODF results. The derived effective temperatures were also checked by fitting the $\mathrm{H} \alpha$ line profile using these models. Special care was taken to achieve a correct continuum fit in the echelle orders containing the $\mathrm{H} \alpha$ line. The typical length of the orders at $6400-6700 \AA$ is 90-100 $\AA$, and we have 2 overlapping orders containing the $\mathrm{H} \alpha$ line. The estimated accuracy of our continuum level is $\pm 1-2 \%$. The $\mathrm{H} \alpha$ profiles of the models are computed as described in Barklem et al. (2000), namely, assuming LTE and using the SYNTH program (Piskunov 1992) with Stark broadening calculations from Stehlé \& Hutcheon (1999) and self-broadening from Barklem et al. (2000). Radiative broadening and an estimate of the pressure broadening by helium are also included. A comparison between the observed and calculated $\mathrm{H} \alpha$ line profiles for HD 32115 is shown in Fig. 1. Plots for HD 37594 show equally satisfactory fits. No atmospheric model can reasonably fit the core of the $\mathrm{H} \alpha$ line in both stars, which is always broader in the observations. Atmospheric parameters finally adopted for the abundance analysis are given in Table 2 .

A further important parameter in the abundance analysis is microturbulent velocity. It was derived from the elements with numerous lines and the most accurate atomic data. Typically the $\xi_{\mathrm{t}}$ value as derived from lines of $\mathrm{Cr}$, $\mathrm{Mn}, \mathrm{Fe}, \mathrm{Ni}$, varies from 2.0 to $2.6 \mathrm{kms}^{-1}$ in HD 32115 , and from 2.2 to $2.8 \mathrm{~km} \mathrm{~s}^{-1}$ in HD 37594 . Ti lines give a systematically higher microturbulent velocity in both stars, up to $3.2 \mathrm{kms}^{-1}$. For abundance calculations we 
Table 2. Atmospheric parameters for HD 32115 and HD 37594 with formal error estimates in units of the last digit in parentheses.

\begin{tabular}{lcccc}
\hline \hline Method & $T_{\text {eff }}$ & $\log g$ & {$[\mathrm{M} / \mathrm{H}]$} & $M_{\mathrm{v}}$ \\
\hline & & HD 32115 & & \\
Photometry & $7300(40)$ & $4.21(01)$ & $0.03(04)$ & $2.84(02)$ \\
Parallax & & $4.24(05)$ & & $2.84(10)$ \\
H $\alpha$ & $7250(100)$ & & & \\
ATLAS9-newODF & 7250 & 4.30 & 0.00 & \\
Adopted & 7250 & 4.20 & & \\
& & HD 37594 & & \\
Photometry & $7170(50)$ & $4.21(03)$ & $-0.15(04)$ & \\
Parallax & & $4.22(05)$ & & \\
H $\alpha$ & $7100(100)$ & & & \\
ATLAS9-newODF & 7170 & 4.25 & -0.30 & \\
Adopted & 7170 & 4.20 & & \\
\hline
\end{tabular}

accepted $\xi_{\mathrm{t}}=2.3 \pm 0.3 \mathrm{~km} \mathrm{~s}^{-1}$ for $\mathrm{HD} 32115$, and $\xi_{\mathrm{t}}=$ $2.5 \pm 0.3 \mathrm{~km} \mathrm{~s}^{-1}$ for HD 37594. These values for turbulent velocity fields in late A stars agree very well with theoretical predictions (Kupka \& Montgomery 2002).

\section{Abundance analysis}

To begin the abundance analysis synthetic spectra are computed for the whole observed region with the adopted atmospheric parameters and solar abundances. All lines that have depths greater than $0.5 \%$ of the continuum flux in the synthetic spectrum were extracted from the VALD database (Kupka et al. 1999). For a few elements it was necessary to make corrections to the atomic line data, and these corrections were made during the final steps of abundance analysis (see sections below for individual elements for details). The spectrum synthesis code SYNTH (Piskunov 1992) was used in all synthetic spectrum calculations. Using these calculations we estimated rotational velocities for both stars, which were found to be $v \sin i=9 \pm 2 \mathrm{~km} \mathrm{~s}^{-1}$ for HD 32115 and $v \sin i=$ $17 \pm 2 \mathrm{~km} \mathrm{~s}^{-1}$ for HD 37594. While for the most part rotational plus instrumental broadening provides adequate fits to unblended spectral lines in HD 32115, this is not the case for HD 37594. Single spectral lines in this star often have V-shaped profiles rather than the usual U-shaped profiles produced by the rotational broadening. Similar line profiles are observed in pulsating stars (e.g. the study of the $\delta$ Sct-type star FG Vir by Mittermayer 2001). This effect is marginal, and needs a further study with higher spectral resolution.

The final abundance analysis was done by three different methods: classical analysis using equivalent width data, spectral synthesis for strong and partially blended lines, and a semi-automatic procedure which uses both equivalent widths and spectrum synthesis techniques. The results of the first two methods were combined. We found no systematic difference between equivalent widths measured in the Solar Flux Atlas (Kurucz et al. 1984) and in a day-sky spectra obtained at the same dates and with the same spectrograph that was used for the taking the spectra of the program stars. The range of the errors in equivalent width measurements is $2-5 \mathrm{~m} \AA$ and depends on $S / N$ ratio (position of the line on the echelle order), and blending effects. For most of the unblended lines this error does not exceed $2-3 \mathrm{~m} \AA$. We also compared two independent sets of equivalent width measurements in the HD 32115 spectrum made by I. Bikmaev with the Gaussian approximation and by H. Bruntt with the Voigt approximation. For 122 lines the mean difference between two sets is 1.2 $\mathrm{m} \AA$ with a standard deviation of $2.8 \mathrm{~m} \AA$.

\subsection{Classical abundance analysis}

Comparing the synthetic and the observed spectra we selected spectral lines that are free of substantial blending effects and measured their equivalent widths. The final abundances were calculated with Kurucz's WIDTH9 code modified by V. Tsymbal for VALD-format input files. For a few elements, like BaII which has mostly very strong lines, or Eu II and Nd III which have only 1-2 partially blended lines in the whole spectral region, abundances were derived with synthetic spectrum calculations. Final abundances for 31 chemical elements (41 ions) are collected in Table 3 (Cols. 2 and 7 for HD 32115 and HD 37594 respectively). The standard deviation is given in units of the last digit in parentheses. Solar abundances in $\log \left(N / N_{\text {tot }}\right)=\log \left(N / N_{\mathrm{H}}\right)-0.04$ (correction due to $\mathrm{He})$ are taken from Grevesse \& Sauval (1998) except C, $\mathrm{N}, \mathrm{O}, \mathrm{Mg}, \mathrm{Si}$, and $\mathrm{Ba}$. For these elements, except Ba, the most recent data by Holweger (2001) are given. We adopted the solar Ba abundance derived by Mashonkina \& Gehren (2000). NLTE corrections were applied for the species marked by asterisks (see details below). Columns 6 and 11 contain the stellar abundance relative to the sun. We also calculated line-by-line differences between stellar and solar abundances, which show a close agreement with the values from Cols. 6 and 11. All individual line abundances are presented in Table 4 which is available 
Table 3. Abundances in HD 32115 and HD 37594 based on $n$ measured lines with the standard deviation in units of the last two digits in parentheses. NLTE corrections are applied for the species marked by asterisks. The letters "H" and "MG" in the last column mean that solar abundances are taken from Holweger (2001) and Mashonkina \& Gehren (2000); otherwise standard solar composition as reported by Grevesse \& Sauval (1998) is used.

\begin{tabular}{|c|c|c|c|c|c|c|c|c|c|c|c|}
\hline \multirow[t]{3}{*}{ Ion } & \multicolumn{5}{|c|}{ HD 32115} & \multicolumn{5}{|c|}{ HD 37954} & \multirow{3}{*}{$\begin{array}{c}\text { Sun } \\
\log \left(N / N_{\text {tot }}\right)\end{array}$} \\
\hline & \multicolumn{2}{|c|}{ Classical analysis } & \multicolumn{2}{|c|}{ Semi-automatic } & \multirow[b]{2}{*}[N/N_{\mathrm{H}}]{} & \multicolumn{2}{|c|}{ Classical analysis } & \multicolumn{2}{|c|}{ Semi-automatic } & \multirow[b]{2}{*}[N/N_{\mathrm{H}}]{} & \\
\hline & $\log \left(N / N_{\text {tot }}\right)$ & $n$ & $\log \left(N / N_{\text {tot }}\right)$ & $n$ & & $\log \left(N / N_{\text {tot }}\right)$ & $n$ & $\log \left(N / N_{\text {tot }}\right)$ & $n$ & & \\
\hline C I & $-3.62(17)$ & 35 & $-3.52(24)$ & 5 & -0.17 & $-3.73(15)$ & 27 & $-3.74(05)$ & 4 & -0.28 & $-3.45 \mathrm{H}$ \\
\hline N I & $-4.19(08)$ & 11 & $-4.22(04)$ & 2 & -0.08 & $-4.27(12)$ & 4 & & & -0.16 & $-4.11 \mathrm{H}$ \\
\hline O I & $-3.23(10)$ & 12 & $-3.25(04)$ & 2 & 0.07 & $-3.36(12)$ & 5 & $-3.27(12)$ & 5 & -0.06 & $-3.30 \mathrm{H}$ \\
\hline Na I* & $-5.86(12)$ & 8 & & & -0.15 & $-6.05(21)$ & 8 & & & -0.34 & -5.71 \\
\hline Mg I & $-4.59(12)$ & 9 & $-4.33(05)$ & 4 & -0.09 & $-4.89(10)$ & 8 & $-4.68(03)$ & 4 & -0.39 & $-4.50 \mathrm{H}$ \\
\hline Mg II & $-4.43(11)$ & 4 & -4.46 & 1 & 0.07 & $-4.79(19)$ & 3 & -4.65 & 1 & -0.29 & $-4.50 \mathrm{H}$ \\
\hline $\mathrm{Al} \mathrm{I}$ & $-5.69(15)$ & 4 & & & -0.12 & $-6.15(06)$ & 2 & & & -0.58 & -5.57 \\
\hline Si I & $-4.53(13)$ & 39 & $-4.49(12)$ & 11 & -0.03 & $-4.75(08)$ & 26 & $-4.76(13)$ & 11 & -0.25 & $-4.50 \mathrm{H}$ \\
\hline Si II & $-4.47(12)$ & 6 & -4.53 & 1 & 0.03 & $-4.59(04)$ & 3 & $-4.72(10)$ & 2 & -0.09 & $-4.50 \mathrm{H}$ \\
\hline P I & $-6.42(13)$ & 2 & & & 0.17 & & & & & & -6.59 \\
\hline S I & $-4.81(15)$ & 19 & $-4.92(09)$ & 5 & -0.10 & $-5.07(10)$ & 8 & $-5.06(01)$ & 2 & -0.36 & -4.71 \\
\hline Cl I & -6.76 & 1 & & & -0.22 & & & & & & -6.54 \\
\hline $\mathrm{K} \mathrm{I}^{*}$ & -7.05 & 1 & & & -0.13 & -7.32 & 1 & & & -0.40 & -6.92 \\
\hline $\mathrm{Ca} \mathrm{I}$ & $-5.63(15)$ & 21 & $-5.60(13)$ & 13 & 0.05 & $-5.89(22)$ & 26 & $-5.99(27)$ & 16 & -0.21 & -5.68 \\
\hline Ca II* & $-5.69(11)$ & 7 & -5.58 & 1 & -0.01 & $-5.89(04)$ & 4 & -5.88 & 1 & -0.21 & -5.68 \\
\hline Sc II & $-8.89(10)$ & 11 & $-8.96(20)$ & 4 & -0.02 & $-9.04(15)$ & 6 & -9.13 & 3 & -0.17 & -8.87 \\
\hline $\mathrm{Ti} \mathrm{I}$ & $-7.20(14)$ & 21 & $-7.18(04)$ & 4 & -0.18 & $-7.43(14)$ & 3 & $-7.42(03)$ & 2 & -0.41 & -7.02 \\
\hline Ti II & $-7.03(15)$ & 27 & $-6.92(20)$ & 13 & -0.01 & $-7.23(18)$ & 19 & $-7.38(12)$ & 8 & -0.21 & -7.02 \\
\hline V I & $-8.24(25)$ & 3 & & & -0.20 & & & & & & -8.04 \\
\hline V II & $-8.17(08)$ & 5 & -7.95 & 1 & -0.13 & $-8.13(25)$ & 2 & & & -0.09 & -8.04 \\
\hline Cr I & $-6.40(12)$ & 39 & $-6.40(13)$ & 7 & -0.03 & $-6.71(10)$ & 11 & $-6.75(06)$ & 5 & -0.34 & -6.37 \\
\hline Cr II & $-6.27(09)$ & 20 & $-6.22(12)$ & 7 & 0.10 & $-6.63(09)$ & 17 & $-6.55(06)$ & 6 & -0.26 & -6.37 \\
\hline Mn I & $-6.87(17)$ & 20 & $-6.76(37)$ & 6 & -0.22 & $-7.14(16)$ & 9 & $-7.04(13)$ & 4 & -0.49 & -6.65 \\
\hline Mn II & $-6.72(11)$ & 2 & & & -0.07 & & & & & & -6.65 \\
\hline Fe I & $-4.60(12)$ & 235 & $-4.59(11)$ & 109 & -0.06 & $-4.88(13)$ & 183 & $-4.89(12)$ & 38 & -0.34 & -4.54 \\
\hline Fe II & $-4.48(14)$ & 60 & $-4.54(11)$ & 23 & 0.06 & $-4.87(11)$ & 25 & $-4.76(11)$ & 4 & -0.33 & -4.54 \\
\hline Co I & $-7.16(10)$ & 5 & $-7.16(00)$ & 2 & -0.04 & $-7.43(03)$ & 3 & & & -0.31 & -7.12 \\
\hline $\mathrm{Ni} \mathrm{I}$ & $-5.91(10)$ & 59 & $-5.94(13)$ & 17 & -0.12 & $-6.24(16)$ & 27 & $-6.18(03)$ & 4 & -0.45 & -5.79 \\
\hline Ni II & -5.82 & 1 & & & -0.03 & & & & & & -5.79 \\
\hline $\mathrm{Cu} \mathrm{I}$ & $-7.82(26)$ & 5 & & & 0.01 & $-8.32(06)$ & 2 & & & -0.49 & -7.83 \\
\hline Zn I & $-7.77(16)$ & 3 & & & -0.33 & $-8.07(05)$ & 3 & & & -0.63 & -7.44 \\
\hline Sr II* & $-9.09(22)$ & 3 & & & -0.02 & $-9.19(07)$ & 3 & & & -0.12 & -9.07 \\
\hline Y II & $-9.68(15)$ & 8 & $-9.74(08)$ & 3 & 0.12 & $-9.94(08)$ & 8 & $-9.95(07)$ & 3 & -0.14 & -9.80 \\
\hline Zr II & $-9.38(16)$ & 7 & & & 0.06 & $-9.42(20)$ & 5 & & & 0.02 & -9.44 \\
\hline Ba II* & $-9.64(12)$ & 6 & $-9.57(05)$ & 3 & 0.19 & $-9.87(28)$ & 4 & $-9.70(19)$ & 4 & -0.04 & $-9.83 \mathrm{MG}$ \\
\hline La II & $-10.82(08)$ & 4 & & & 0.05 & $-11.15(13)$ & 3 & & & -0.28 & -10.87 \\
\hline Ce II & $-10.41(19)$ & 7 & & & 0.05 & $-10.57(16)$ & 6 & & & -0.11 & -10.46 \\
\hline Nd II & $-10.49(25)$ & 11 & & & 0.05 & $-10.63(01)$ & 2 & & & -0.09 & -10.54 \\
\hline Nd III & $-10.40(07)$ & 2 & & & 0.14 & & & & & & -10.54 \\
\hline Sm II & -11.06 & 1 & & & -0.03 & -11.38 & 1 & & & -0.35 & -11.03 \\
\hline Eu II & -11.63 & 1 & & & -0.10 & -11.80 & 1 & & & -0.27 & -11.53 \\
\hline
\end{tabular}


in electronic form only. This table may be retrieved from

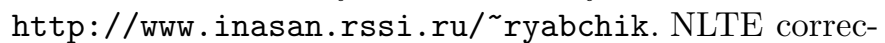
tions are also included in Table 4 . These are probably the most complete abundance data for single A-type stars to date.

\subsection{Semi-automatic abundance analysis}

We have written software called vwA for semi-automatic abundance analysis which is described in Bruntt et al. (2002). The code runs though the list of atomic line data extracted from VALD (Kupka et al. 1999) for the target star, and selects lines that suffer from the least amount of blending. For a region around each selected line the synthetic spectrum is calculated for different abundances of the element forming the line until the equivalent widths of the observed and synthetic spectrum match. The computation of the synthetic spectrum is done with SYNTH version 2.5 (Valenti \& Piskunov 1996). The code needs the line data from VALD and the adopted atmosphere model as input. When vwA has fitted the selected lines they are inspected visually - and the fit is either accepted, rejected or must be improved manually.

While classical abundance analysis can be performed relatively fast for a star like HD 32115 that has low $v \sin i$ the advantage of using vwA for abundance analysis is demonstated for HD 37594. Since this star has a moderate value of $v \sin i$ one needs to select lines with some amount of blending to be able to derive abundances of as many elements as possible. We first used vwA where we only selected unblended Fe lines. We then adjusted the microturbulence and recalculated abundances with VWA until the Fe I abundance and equivalent width of the lines did not correlate. We thus found $\xi_{\mathrm{t}}=2.4 \pm 0.3 \mathrm{~km} \mathrm{~s}^{-1}$. Since the abundance of Fe was now considered to be accurate, we allowed VWA to select lines of other elements that were mild blends with Fe. By this method we were able to use lines that could not be used in the classical abundance analysis which is strictly limited to unblended lines.

When comparing the results of the abundance analysis of the two methods in Table 3 we see that far fewer lines are chosen by VWA but the derived abundances all agree within the error estimates. Hence, vwA is usable for carrying out fast and reliable abundance analysis.

\subsection{Errors in abundance determinations}

Our derived abundances contain errors from a number of sources of uncertainty, including the adopted atomic data, most importantly the oscillator strengths, the equivalent width measurements, the adopted atmospheric parameters and model, and the observed spectra. For some sources we can directly estimate the associated error. The estimated uncertainty in equivalent width measurements is $\pm 2 \mathrm{~m} \AA$, which results in $0.20-0.09$ dex error in the abundance for lines with equivalent widths 5 to $10 \mathrm{~m} \AA$, $0.05 \mathrm{dex}$ for $W_{\lambda}=20 \mathrm{m \AA}$, and less than 0.03 dex for $W_{\lambda} \geq 40 \mathrm{~m} \AA$. An error of $100 \mathrm{~K}$ in the effective temperature determination results in a systematic error of 0.05 dex on the the abundance of the most temperature-sensitive atomic species, e.g. Ti I, V I. For cases where a suitably large number of lines are used, the standard deviations quoted in Table 3 estimate the total random error associated with all sources of uncertainty. However, for species where only a small number of lines are available this cannot be expected to give any indication of this error. One can see from Table 3 that the standard deviations for most elements with a sufficient number of spectral lines do not exceed 0.15 dex. Considering this as indicative for all elements, and taking into account also possible systematic errors we estimate the total error at about 0.2 dex for the species with 1-3 spectral lines.

Formally, a gravity decrease by 0.2 dex may provide ionization balance for all iron peak elements, since abundances obtained from the ionized lines will be decreased by $0.06-0.09$ dex, while abundances from the neutral lines would not be changed. CNO abundances would be reduced by 0.05 dex. However, we stress that the effective gravity for both stars is accurately defined by parallax measurements.

Since most solar photospheric abundances are derived with the semi-empirical model of Holweger \& Müller (1974), it is important to check that our use of theoretical Kurucz models does not itself introduce any significant differences relative to the solar abundances. To test this we compared solar Fe abundances derived from Fe II lines in the solar spectrum employing the HolwegerMüller solar atmospheric model (Holweger \& Müller 1974) with those found using the solar model computed with ATLAS9 and the same convection treatment as for our stars (see Gardiner et al. 1999). The abundances were calculated from the best 13 Fe II lines (Schnabel et al. 1999) with oscillator strengths from their paper and from the VALD database. The results are: $\log N_{\mathrm{Fe}}=7.42 \pm$ 0.09 (SKH $\log g f$ values, HM model, weighted mean), $\log N_{\mathrm{Fe}}=7.46 \pm 0.11$ (SKH $\log g f$ values, our model, unweighted mean), $\log N_{\mathrm{Fe}}=7.48 \pm 0.10$ (VALD $\log g f$ values, our model, unweighted mean). With our solar model the line-by-line difference (HD 32115-Sun) for 31 common Fe II lines is $-0.02 \pm 0.10$ and agrees within the error limits with the $[\mathrm{Fe} / \mathrm{H}]$ value presented in Table 3 .

\subsection{CNO elements}

Oscillator strengths for $\mathrm{C}$ and $\mathrm{N}$ were taken from Hibbert et al. (1993) and Hibbert et al. (1991) respectively. The same data were used in reevaluation of the solar abundances (Holweger 2001). For oxygen, we employed the oscillator strengths supplied by VALD which originate from the NIST compilation (Wiese et al. 1996). We did not use the IR O I triplet because of the possibly strong NLTE effects. Expected NLTE corrections are -0.1 dex for C (see Sturenburg 1993; Rentzsch-Holm 1996; 
Paunzen et al. 1999) and $-0.01--0.03$ dex for O (Takeda 1997). Both stars show similar small deficiencies of $\mathrm{C}$ and N.

\subsection{Na to $K$}

These elements are known to be subject to NLTE effects. We also note that the oscillator strengths for Si I lines in VALD are not accurate, many of them coming from Kurucz \& Peytremann (1975). Therefore we recalculated oscillator strengths for most of the $\mathrm{Si}$ I lines by fitting them to the Solar Flux Atlas (Kurucz et al. 1984) adopting the solar abundance in Table 3. NLTE corrections were calculated for $\mathrm{NaI}$, and $\mathrm{K}$ I following atomic models and procedures developed by Mashonkina et al. (2000) for $\mathrm{Na}$, and Ivanova \& Shimansky (2000) for K. Oscillator strength data for $\mathrm{Al}$ I were taken from Baumüller \& Gehren (1996). According to this paper NLTE corrections for the $\mathrm{Al}$ I lines used in our abundance analysis are negligible. For $\mathrm{Mg}$ I lines $\lambda \lambda$ 4703, 4730, 5528, 5711 oscillator strengths were taken from Jonsson et al. (1984) and from Froese Fischer (1975). In both stars the Mg abundance was derived by the spectral synthesis technique with the Stark and Van der Waals damping constants giving the best fit for the same Mg I lines in the spectra of Procyon and the Sun (Fuhrmann et al. 1997; Zhao et al. 1998). Individual LTE and NLTE abundances are given in Table 4. The largest NLTE corrections were found for the K I resonance line $\lambda$ 7698.97. They are -0.61 (HD 32115) and -0.41 (HD 37594). Unfortunately, another K I resonance line at $\lambda 7664.91$ falls in one of the gaps in our echelle spectra. We could measure the strongest $\mathrm{Cl}$ I line $\lambda 8375.95$ in the spectrum of HD 32115 . The solar photospheric $\mathrm{Cl}$ abundance is very uncertain, $\log \left(\mathrm{Cl} / N_{\text {tot }}\right)=$ $-6.54 \pm 0.3$. The $\mathrm{Cl}$ abundance in HD 32115 was obtained from just one weak line but agrees perfectly with the $\mathrm{Cl}$ abundance found in meteorites (Grevesse \& Sauval 1998). Two strongest infrared $P$ I lines $\lambda \lambda$ 9593.50, 9796.83 measured in the latest spectrum of HD 32115 were used to derive phosphorus abundance.

\subsection{Ca to $\mathrm{Ni}$}

The iron-group elements usually represent the metallicity of a star. With a few exceptions we were able to obtain abundances from the lines of neutral and singly-ionized species for all elements of this group, thus checking the ionization balance in the atmospheres. The abundances obtained by the neutral and ionized Fe-group elements differ by $\approx 0.13$ dex on average. In both our stars the Fe-group elements in the ionized state are dominating and therefore their lines are less sensitive to temperature inaccuracy and NLTE effects and hence should provide better abundance estimates. Among iron-group elements NLTE calculations were carried out for $\mathrm{Ca}$ (see below) and Fe (Rentzsch-Holm 1996). An expected NLTE correction to the abundance obtained from Fe I lines is $\approx+0.1$ dex for $T_{\text {eff }} \approx 7300 \mathrm{~K}$.

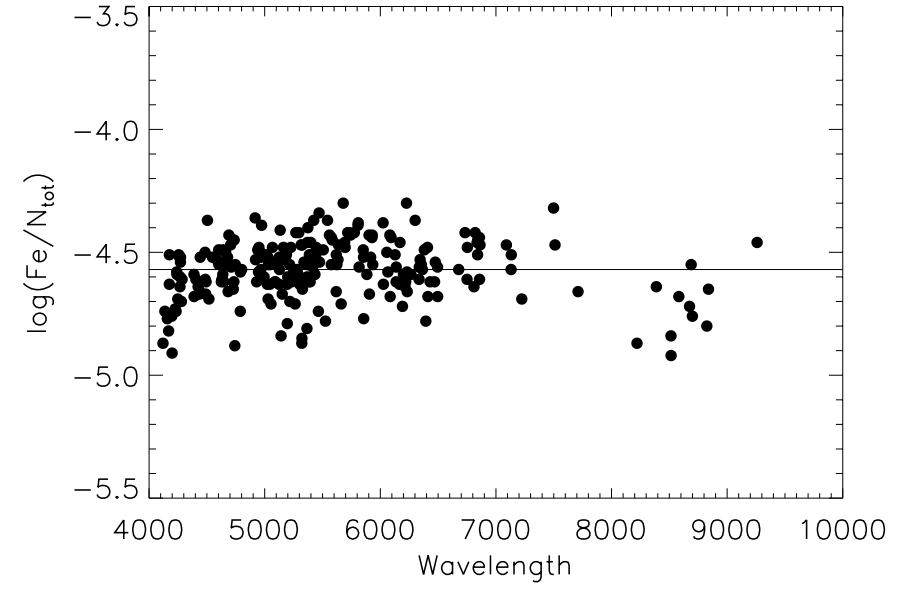

Fig. 2. The dependence of the individual abundances obtained from Fe I lines in HD 32115 on wavelength.

Applying this correction as a first approximation to the abundances obtained from the lines of neutral elements a reasonable ionization balance is obtained.

Five Fe II lines correspondning to $4 \mathrm{~d}-4 \mathrm{f}$ transitions with an upper energy level at around $10.5 \mathrm{eV}$ were measured in the HD 32115 spectrum. For these lines only theoretical oscillator strengths are available. Both the $\log g f$ values from the Kurucz line list (included in VALD) (Kurucz 1993) and the more accurate sets of calculated $\log g f$-values from Raassen \& Uylings (1998) agree to within 0.07 dex. Abundances obtained from these lines do not deviate by more than $0.1-0.2$ dex from the mean Fe abundance and thus supports the oscillator strengths for these high excitation Fe II lines to this level of accuracy.

Fe abundance analysis in HD 32115 is based on about 300 lines well spread through the whole spectral region of 4100-9200 $\AA$. We did not find any clear dependence of the individual abundances on the wavelength (Fig. 2) which might be expected in the primary star if the contribution from the secondary is not negligible.

For the two strongest Ca II lines $\lambda \lambda$ 8912, 8927 we increased $\log g f$ by 0.3 dex to fit line profiles in the solar spectrum. Stark damping constants for these lines, which are important, are taken from Dimitrijević \& Sahal-Bréchot (1993). We also carried out NLTE calculations for Ca II lines using a model of the atom developed by Ivanova et al. (2002). NLTE corrections are always negative and of the order of -0.15 dex for $\lambda \lambda 8248,8912,8927$ lines in both stars, while they are negligible for weak Ca II lines.

\section{7. $\mathrm{Cu}$ and $\mathrm{Zn}$}

Abundances of these elements were obtained by spectral synthesis taking into account hyperfine splitting (hfs). Hfs constants were extracted from Biehl (1976). Solar synthetic spectrum calculations showed that for $\mathrm{Zn}$ I lines we may neglect the hfs effect, while it is strong for $\mathrm{Cu}$ I lines in the solar spectrum. In both our stars $\mathrm{Cu}$ I lines are weak, and the hfs effect for $\mathrm{Cu}$ I and $\mathrm{Zn}$ I lines is not significant. 
Zn shows the largest deviation from the solar abundance in HD 32115, and the largest deficiency in HD 37594. To our knowledge, there are no NLTE calculations for $\mathrm{Cu}$ I or $\mathrm{ZnI}$. With ionization energies $\chi=$ $7.72 \mathrm{eV}$ for $\mathrm{Cu} \mathrm{I}$ and $9.39 \mathrm{eV}$ for $\mathrm{ZnI}$ these atoms are minor species similar to $\mathrm{Mg}$ I, Al I, Fe I in the stellar atmospheres typical for investigated stars. Based on our experience in NLTE analyses and taking into account NLTE calculations for atoms with similar atomic parameters and term structures (Sturenburg 1993; Rentzsch-Holm 1996) $\mathrm{Cu}$ I and $\mathrm{ZnI}$ are expected to be over-ionized. In this case low atomic levels are underpopulated compared with LTE, and the lower the excitation energy is the greater the departures from LTE are. Most probably, NLTE effects will weaken the investigated $\mathrm{Cu}$ I and $\mathrm{Zn}$ I lines and NLTE abundance corrections are expected to be positive. Usually NLTE corrections are increasing with the line intensity. The observed equivalent widths of $\mathrm{Zn}$ I lines are 2-3 times larger than for $\mathrm{Cu}$ I lines, therefore we may also expect larger positive NLTE corrections for Zn.

\section{8. $\mathrm{Sr}, \mathrm{Y}, \mathrm{Zr}, \mathrm{Ba}$}

NLTE corrections for Sr II lines were calculated using the model atom developed by Belyakova et al. (1997, 1999). They do not exceed 0.1 dex. Hfs effects may be important for Y II lines, but there are no data available. Zr abundances are rather uncertain, because reliable oscillator strengths are only known for a small number of spectral lines. We could not use the solar spectrum fitting for Zr II lines measured in our stars due to strong molecular contributions in the corresponding spectral regions.

NLTE corrections to the Ba abundance were calculated according to Mashonkina et al. (1999). They are large, up to 0.18 dex, for the two strongest Ba II lines $\lambda \lambda 6141,6496$. It should be mentioned that the solar Ba abundance used by us was derived by Mashonkina \& Gehren (2000) from the same lines as in our analysis, and it is closer to abundance of $\mathrm{Ba}$ found in meteorites than any previously published solar Ba abundance values (see Grevesse \& Sauval 1998). Stark damping constants for Ba II lines were taken from Dimitrijević \& Sahal-Bréchot (1996), while for resonance Sr II lines the corresponding values were taken from the NIST compilation (Konjević et al. 1984).

\subsection{La to Eu}

New experimental oscillator strengths and hfs constants were used for La II (Lawler et al. 2001a), Ce II (Palmeri et al. 2000; Zhang et al. 2001), and Eu II lines (Lawler et al. 2001b). Two weak lines of Nd III $\lambda \lambda$ 5102, 5295, which are the strongest lines in the Nd III spectrum, were identified in HD 32115. They are partially blended so abundance estimates were made by spectral synthesis. Oscillator strengths and other atomic parameters in VALD are taken from Cowley \& Bord (1998) and from Bord (2000). Both lines provide Nd abundances close to that

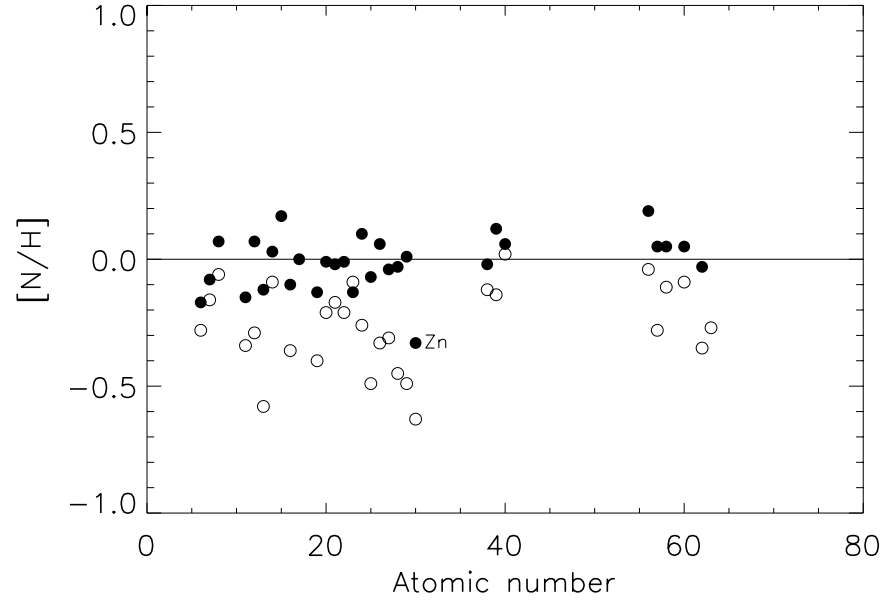

Fig. 3. The observed relative abundances in HD 32115 (filled circles) and in HD 37594 (open circles).

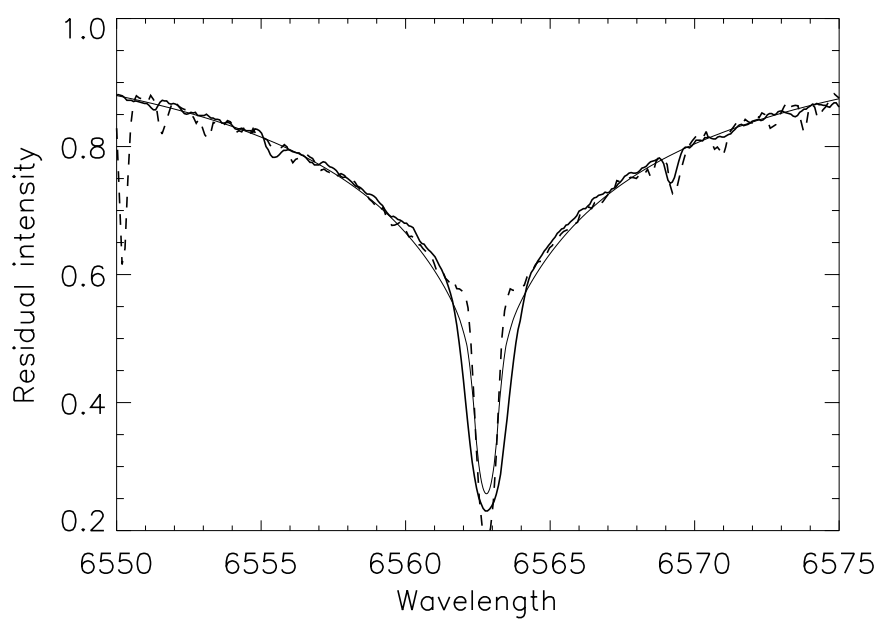

Fig. 4. A comparison between $\mathrm{H} \alpha$ line profiles observed in HD 32115 (thick full line) and in roAp star HD 24712 (dashed line), and calculated for the adopted 7250-ODF model (thin full line).

obtained from the analysis of Nd II lines, thus giving strong support for the Nd III oscillator strength calculations.

\section{Discussion}

The abundances found for HD 32115 and HD 37594 relative to the sun are shown in Fig. 3 by filled and open circles respectively. Within the typical error limits of \pm 0.15 dex HD 32115 is a solar abundance star (a mean metallicity $[M]=-0.04 \pm 0.11)$. Therefore this star may be used as a chemical standard in studies of cool peculiar stars. We also get a standard for further investigation of the hydrogen wing-to-core anomaly found in cool Ap stars by Cowley et al. (2001). Figure 4 shows a comparison between $\mathrm{H} \alpha$ line profiles in HD 32115 and in one of the pulsating Ap (roAp) stars, HD 24712, with the same effective temperature. It is evident from this comparison that the Ap-star anomaly occurs in that part of the hydrogen line core that is not reproduced by our current models. 
HD 37594 is slightly metal-deficient. Its mean metallicity $[M]=-0.26 \pm 0.16$ is close to the value $[M]=-0.15 \pm$ 0.04 obtained from photometric calibrations. This error in the metallicity obtained from photometric calibrations is based only on the errors in the photometric indices. No systematic errors of the calibration itself are included, which may be up to 0.1. Further support for the high accuracy of the metallicities derived from the Strömgren photometry for normal stars is provided by the results of abundance analysis of 28 And=HD 2628 (Adelman et al. 2000) and $\sigma$ Boo A=HD 128167 (Adelman et al. 1997). $[\mathrm{M}]=-0.16 \pm 0.29$ and $-0.29 \pm 0.37$ were derived for these stars from abundances versus -0.13 and -0.35 from photometric calibration. The abundance pattern in HD 37594 is similar to that in 28 And $\left(T_{\text {eff }}=7250 \mathrm{~K}\right.$, $\log g=4.2, \xi_{\mathrm{t}}=2.3 \mathrm{~km} \mathrm{~s}^{-1}, v \sin i=9 \mathrm{~km} \mathrm{~s}^{-1}-$ see Adelman et al. 2000) which belongs to the $\delta$ Sct group. The smaller scatter derived in the present paper is explained by more accurate abundance determinations with NLTE and hfs effects taken into account as well as more careful choice of the unblended lines which is possible in the red spectral region.

Abundances obtained for HD 32115 and HD 37594 do not follow the predicted abundance pattern for $Z \leq 28$ from consistent stellar evolution models calculated with radiative forces, opacities and diffusion (Turcotte et al. 1998). Models for $1.5 \mathrm{M} / \mathrm{M}_{\odot}$ predict that elements with $5<Z<20$ will be underabundant by 0.5 dex relative to $\mathrm{Fe}$, and that iron-peak elements will be generally slightly overabundant even at early evolutionary phases on the MS. This was not observed in either of the stars analysed here.

To confirm the derived abundances we have used the software VwA (Bruntt et al. 2002) which was developed to be able to make fast semi-automatic abundance analysis. We find the same abundances as the more careful classical approach. At present VWA is being applied to the study of the primary target candidate stars for the asteroseismology missions COROT and Rømer (Bruntt et al. 2002). Several of these stars have moderate or high $v \sin i$ and the amount of work needed to carry out the analysis using VWA is reduced substantially.

Acknowledgements. We thank G. Wade who provided us with the spectrum of $\mathrm{HD} 24712$ in the $\mathrm{H} \alpha$ region, A. Bondar for the help during the observations, N. Sakhibullin for useful discussions, and F. Kupka for his help in new model atmosphere calculations. T. R. thanks the Fonds zur Förderung der wissenschaftlichen Forschung (project $P-14984$ ), the Jubiläumsfonds der Österreichischen Nationalbank (project 7650), the Russian National Program "Astronomy", and RFBR (grant 00-15-96722) for financial support. IFB, LIM. EVB, VVS acknowledge the Russian National Program "Astronomy", and RFBR (grant 02-02-17174). FAM and GG thank the Russian National Program "Astronomy", and RFBR (grant 02-02-17423) for partial funding. The authors thank the referee, C.R. Cowley, for his helpful comments.

\section{References}

Abt, H. A., \& Morrell, N. I. 1995, ApJS, 99, 135

Adelman, S. J., Caliskan, H., Kocer, D., Cay, I. H., \& Tektunali, H. G. 2000, MNRAS, 316, 514

Adelman, S. J., Caliskan, H., Kocer, D., \& Bolcal, C. 1997, MNRAS, 288, 470

Barklem, P. S., Piskunov, N., \& O'Mara, B. J. 2000, A\&A, 363, 1091

Baumüller, D., \& Gehren, T. 1996, A\&A, 307, 961

Belyakova, E., \& Mashonkina, L. I. 1997, Astron. Rep., 41, 530

Belyakova, E., Mashonkina, L. I., \& Sakhibullin, N. A. 1999, Astron. Rep., 43, 819

Biehl, D. 1976, Ph.D. Thesis, Christian-Albrechts-Universität, Kiel, Institute für Theoretische Physik und Sternwarte

Bord, D. 2000, A\&AS, 144, 517

Bruntt, H., Catala, C., Garrido, R., et al. 2002, A\&A, 389, 345

Cowley, A., Cowley, C., Jaschek, M., \& Jaschek, C. 1969, AJ, 74,375

Cowley, C. R., \& Bord, D. J. 1998, in The Scientific Impact of the Goddard High Resolution Spectrograph, ed. J. C. Brandt, T. B. Ake, \& C. C. Peterson, ASP Conf. Ser., 143, 346

Cowley, C. R., Hubrig, S., Ryabchikova, T. A., et al. 2001, A\&A, 367, 939

Dimitrijević, V. S., \& Sahal-Bréchot, S. 1993, JQSRT, 49, 157

Dimitrijević, V. S., \& Sahal-Bréchot, S. 1996, 18th SPIG Summer School and International Symposium on the Physics of Ionized Gases, Contributed papers \& abstracts of invited lectures and progress reports, Yugoslavia, 548

ESA. 1997, The Hipparcos and Tycho catalogues, ESA SP-1200

Froese Fischer, C. 1975, Can. J. Phys., 53, 184

Fuhrmann, K., Pfeiffer, M., Frank, C., Reetz, J., \& Gehren, T. 1997, A\&A, 323, 909

Galazutdinov, G. A. 1992, SAO RAS Preprint No. 92

Gardiner, R. B., Kupka, F., \& Smalley, B. 1999, A\&A, 347, 876, 1999

Grenier, S., Burnage, R., Farraggiana, R., et al. 1999, A\&AS, 135,503

Grevesse, N., \& Sauval, A. J. 1998, Sp. Sci. Rev., 85, 161

Gustafsson, B. 1998, Sp. Sci. Rev., 85, 419

Hauck, B., \& Mermilliod, M. 1998, A\&AS, 129, 431

Hibbert, A., Biémont, E., Godefroid, M., \& Vaeck, N. 1991, A\&AS, 88, 505

Hibbert, A., Biémont, E., Godefroid, M., \& Vaeck, N. 1993, A\&AS, 99, 179

Holweger, H. 2001, in Solar and Galactic Composition, ed. R. F. Wimmer-Schwengruber, AIP Conf. Proc., 598, 23

Holweger, H., \& Müller, E. A. 1974, Solar Phys., 39, 19

Ivanova, D. V., Sakhibullin, N. A., \& Shimansky, V. V. 2002, Astron. Rep., 46, 390

Ivanova, D. V., \& Shimansky, V. V. 2000, Astron. Rep., 44, 376

Jonsson, G., Kroll, S., Persson, A., \& Svanberg, S. 1984, Phys. Rev. A, 30, 2429

Konjević, N., Dimitrijević, V. S., \& Wiese, W. L. 1984, J. Phys. Chem. Ref. Data, 13, 649

Kupka, F., Piskunov, N., Ryabchikova, T. A., Stempels, H. C., \& Weiss, W. W. 1999, A\&AS, 138, 119

Kupka, F., \& Momtgomery, M. H. 2002, MNRAS, in press

Kurucz, R. L., \& Peytremann, E. 1975, SAO Spec. Rep., No. 362 
Kurucz, R. L., Furenlid, I., Brault, J., \& Testerman, L. 1984, NSO Atlas No. 1: Solar Flux Atlas from 296 to $1300 \mathrm{~nm}$, Sunspot, NSO

Kurucz, R. L. 1993, CD-ROM's 18-23

Lawler, J. E., Bonvallet, G., \& Sneden, C. 2001a, ApJ, 556, 452

Lawler, J. E., Wickliffe, M. E., Den Hartog, E. A., \& Sneden, C. 2001b, ApJ, 563, 1075

Luck, R. E., \& Lambert, D. L. 1981, ApJ, 245, 1018

Luck, R. E., \& Lambert, D. L. 1985, ApJ, 298, 782

Mashonkina, L., \& Gehren, T. 2000, A\&A, 364, 249

Mashonkina, L., Gehren, T., \& Bikmaev, I. 1999, A\&A, 343, 519

Mashonkina, L. I., Shimansky, V. V., \& Sakhibullin, N. A. 2000, Astron. Rep., 44, 790

Mittermayer, P. 2001, Die Atmosphäre des $\delta$ Scuti Sterns FG Virginis, Master Thesis, University of Vienna

Moon, T. T., \& Dworetsky, M. M. 1985, MNRAS, 217, 305

Musaev, F. A., Galazutdinov, G. A., Sergeev, A. V., Karpov, N. V., \& Pod'yachev, Yu. V. 1999, Kinematics and physics of celestial bodies, 13, 282 (in Russian)

Napiwotzki, R., Schönberner, D., \& Wenske, V. 1993, A\&A, 268,653

Nave, G., Johansson, S., Learner, R. C. M., Torne, A. P., \& Brault, J. W. 1994, ApJS, 94, 221

Nordström, B., \& Andersen, J. 1985, A\&AS, 61, 53

Palmeri, P., Quinet, P., Wyart J.-F., \& Biémont, E. 2000, Phys. Scr., 61,323

Pamyatnykh, A. A. 2000, Delta Scuti and Related Stars, ed. M. Breger, \& M. H. Montgomery, ASP Conf. Ser., 210, 215
Pamyatnykh, A. A., Dziembowski, W. A., Handler, G., \& Pikall, H. 1998, A\&A, 333, 141

Paunzen, E., Kamp, I., Iliev, I. Kh., et al. 1999, A\&A, 345, 597

Piskunov, N. 1992, Stellar Magnetism - Proceedings of the international meeting on the problem Physics and Evolution of Stars, ed. Yu. V. Glagolevskij, \& I. I. Romanyuk, St. Petersburg, 92

Piskunov, N., \& Kupka, F. 2001, ApJ, 547, 1040

Raassen, A. J. J., \& Uylings, P. H. M. 1998, A\&A, 340, 300

Rentzsch-Holm, I. 1996, A\&A, 312, 966

Rogers, N. Y. 1995, Comm. in Asteroseismology, No. 78

Schnabel, R., Kock, M., \& Holweger, H. 1999, A\&A, 342, 610 Smalley, B. 1993, A\&A, 274, 391

Smalley, B., \& Kupka, F. 1997, A\&A, 328, 349

Stehlé, C., \& Hutcheon, R. 1999, A\&AS, 140, 93

Sturenburg, S. 1993, A\&A, 277, 139

Takeda, Y. 1997, PASJ, 49, 471

Takeda, Y., \& Takada-Hidai, M. 1998, PASJ, 50, 629

Turcotte, S., Richer, J., \& Michaud, G. 1998, ApJ, 504, 559

Valenti, J. A., \& Piskunov, N. 1996, A\&AS, 118, 595

Varenne, O., \& Monier, R. 1999, A\&A, 351, 247

Wiese, W. L., Fuhr, J. R., \& Deters, T. M. 1996, Atomic Transition Probabilities of Carbon, Nitrogen and Oxygen: A critical data compilation, J. Phys. Chem. Ref. Data, Mono. 7

Zhang, Z. G., Svanberg, S., Jiang, Z., et al. 2001, Phys. Scr., 63,122

Zhao, G., Butler, K., \& Gehren, T. 1998, A\&A, 333, 219 